\title{
Ultrafast-laser dicing of thin silicon wafers: strategies to improve front- and backside breaking strength
}

\author{
Matthias Domke ${ }^{1} \cdot$ Bernadette Egle $^{2} \cdot$ Sandra Stroj $^{1} \cdot$ Marius Bodea $^{3} \cdot$ \\ Elisabeth Schwarz ${ }^{3}$ Gernot Fasching ${ }^{4}$
}

Received: 11 September 2017 / Accepted: 31 October 2017 / Published online: 8 November 2017

(c) The Author(s) 2017. This article is an open access publication

\begin{abstract}
Thin 50- $\mu \mathrm{m}$ silicon wafers are used to improve heat dissipation of chips with high power densities. However, mechanical dicing methods cause chipping at the edges of the separated dies that reduce the mechanical stability. Thermal load changes may then lead to sudden chip failure. Recent investigations showed that the mechanical stability of the cut chips could be increased using ultrashort-pulsed lasers, but only at the laser entrance (front) side and not at the exit (back) side. The goal of this study was to find strategies to improve both front- and backside breaking strength of chips that were cut out of an 8 " wafer with power metallization using an ultrafast laser. In a first experiment, chips were cut by scanning the laser beam in single lines across the wafer using varying fluencies and scan speeds. Three-point bending tests of the cut chips were performed to measure front and backside breaking strengths. The results showed that the breaking strength of both sides increased with decreasing accumulated fluence per scan. Maximum breaking strengths of about $1100 \mathrm{MPa}$ were achieved at the front side, but only below $600 \mathrm{MPa}$ were measured for the backside. A second experiment was carried out to optimize the backside breaking strength. Here, parallel line scans to
\end{abstract}

Matthias Domke

matthias.domke@fhv.at

1 Josef Ressel Center for Material Processing with Ultrashort Pulsed Lasers, Research Center for Microtechnology, Vorarlberg University of Applied Sciences, Hochschulstraße 1, 6850 Dornbirn, Austria

2 Hirschmann Automotive GmbH, Oberer Paspelsweg 6-8, 6830 Rankweil, Austria

3 Infineon Technologies Austria AG, Siemensstr. 2, 9500 Villach, Austria

4 ams AG, Tobelbaderstr., Premstätten, Austria increase the distance between separated dies and step cuts to minimize the effect of decreasing fluence during scribing were performed. Bending tests revealed that breaking strengths of about $1100 \mathrm{MPa}$ could be achieved also on the backside using the step cut. A reason for the superior performance could be found by calculating the fluence absorbed by the sidewalls. The calculations suggested that an optimal fluence level to minimize thermal side effects and periodic surface structures was achieved due to the step cut. Remarkably, the best breaking strengths values achieved in this study were even higher than the values obtained on state of the art ns-laser and mechanical dicing machines. This is the first study to the knowledge of the authors, which demonstrates that ultrafast-laser dicing improves the mechanical stability of thin silicon chips.

\section{Introduction}

Miniaturization and performance enhancement of consumer electronic products drive the development of new emerging chip fabrication technologies. Thinned silicon wafers with thicknesses of about $50 \mu \mathrm{m}$ enable higher packaging densities for three-dimensional (3D) silicon integration and reduce the heat resistance to improve heat dissipation for increasingly powerful chips [1,2]. Moreover, thinning increases the mechanical flexibility of the wafers, which enables the utilization of thin silicon wafers for bendable and flexible devices [3, 4]. However, the reduction of the wafer thickness causes new problems for established dicing methods.

Mechanical dicing becomes more challenging because the diamond blades of the saw cannot resharpen themselves at the thin wafer edges in the range of $100 \mu \mathrm{m}$ or less [5]. Moreover, mechanical dicing causes chippings at the edges 
$[6,7]$ that reduce the mechanical stability of the chip. Thermal load changes may then lead to sudden chip failure [8]. The fracture strength of a mechanically diced wafer with a thickness of $50 \mu \mathrm{m}$ is about $666 \mathrm{MPa}$ [6]. Since laser dicing is a contact-free method, chippings at the edges can be avoided. Consequently, the challenges for laser dicing are to improve the mechanical stability of the cut chips and to achieve the cutting speed of a wafer saw, which is in the order of $100 \mathrm{~mm} / \mathrm{s}$ [9].

The highest edge quality provide laser dicing methods based on thermal stress generation $[10,11]$ or stealth dicing [12]. In both cases, wavelength, pulse duration, and focusing conditions are typically chosen such that the absorption takes place mainly inside the volume of the wafer $[10,13]$. In the first example, the laser acts as volume heat source that generates a stress field which causes the wafer to crack [10, $11]$. In the case of stealth dicing [12, 13], pulsed laser light is focused tightly below the surface to generate a defect inside the volume. To separate the dies, the crack or the defects can be guided by translating the beam across the wafer. In this way, breaking strengths of above $1100 \mathrm{MPa}$ were achieved for separating wafers with a thickness of $220 \mu \mathrm{m}$ [10]. Unfortunately, these methods have the following limitations. The laser tracks must be free from front-side metallization to avoid absorption at the surface. Backside metallization may hold the chips together after laser processing. Scratches at the rough backside, which originate from grinding, may change crack direction or cause additional paths. To overcome these limitations, a full cut is the only solution.

One of the main factors influencing cutting quality is the pulse duration of the laser source. If the pulse duration is reduced down to the fs-regime, the thermal penetration depth becomes equal to the optical penetration depth, and thermal side effects reduce to a minimum [14]. Because the mechanical stability of laser-cut dies is related to the size of the heat-affected zone [15], it can be also improved by reducing the pulse duration, which was already demonstrated in several bending test experiments $[8,10,15]$. Remarkably, the breaking strengths of ps- of fs-laser-cut chips are very high at the laser entrance side [10,15], even higher than those of mechanically diced chips [10], but about a factor of two lower at the laser exit side [10]. This could be related to the following phenomena. Self-organizing periodic holes arise during scribing of $\mathrm{Si}[16,17]$, which cause periodic kerfs at the lower sidewalls and backside after cutting the chips. The kerfs act as stress concentrators, which reduces the breaking strength of the chip backside $[6,8,18]$. The major challenge for ultrashort pulse laser dicing is to find the right parameters, which inhibit these kerfs and increase the mechanical stability of the cut chips at the laser exit side.

In this study, the influence of fluence, scan speed, and number of scans on the front and backside strength was investigated, different strategies to improve the backside strength were tested, and the best results were compared to ns-laser and mechanically diced wafers. To the knowledge of the authors, this is the first study that demonstrates that fs-laser-dicing improves the breaking strength on the front and backside of chips compared to mechanical dicing.

\section{Materials and methods}

The laser dicing experiments were carried out on metallized 8 " Si wafers mounted on a polymer dicing tape. The layer stack from bottom to top consisted of backside metallization $(\approx 600 \mathrm{~nm}), \mathrm{Si}(\approx 60 \mu \mathrm{m})$, frontside power metallization $(\approx 5 \mu \mathrm{m})$, and a water-soluble protective film (HogoMax, Disco). The chips had a width of $w \approx 3 \mathrm{~mm}$ and a length of $b \approx 4 \mathrm{~mm}$.

The absorption of silicon increases towards shorter wavelengths and due to non-linear absorption processes also towards shorter pulse durations. Regarding the wavelength, UV-laser light is absorbed most efficiently, but such systems are also more expensive, have higher conversion losses and thus lower powers, and are more prone to degradation. For these reasons, the laser wavelength was selected such that the photon energy was at least higher than the band gap of silicon to enable direct absorption. Ti: sapphire lasers fulfill this criteria and provide also the shortest pulse durations. However, these lasers typically emit pulses with energies of several $100 \mu \mathrm{J}$, but only at frequencies of a few $\mathrm{kHz}$. To minimize the width of the dicing lines, the focus radius should be in the order of $10 \mu \mathrm{m}$. Thus, lower pulse energies are sufficient to achieve optimal fluences for efficient ablation, and higher pulse frequencies are favored for higher throughput. For this reason, an ultrafast laser (Spirit, Spectra-Physics, Rankweil, Austria) with the following specifications was selected for this study. The pulse duration was about $380 \mathrm{fs}$ (FWHM). The laser could be operated at a wavelength of $\lambda=1040 \mathrm{~nm}$ or $\lambda=520 \mathrm{~nm}$. Because of the direct absorption of silicon at the latter wavelength, all experiments in this study were carried out at $\lambda=520 \mathrm{~nm}$. The laser source could be operated at a pulse frequency between $200 \mathrm{kHz}$ and $1 \mathrm{MHz}$. All experiments were carried out using a pulse repetition rate of $f_{\text {rep }}=200 \mathrm{kHz}$, because the laser system provided maximum pulse energy and power at this pulse frequency. The maximum power and pulse energy available behind the focusing optics were measured to be $P=1.6 \mathrm{~W}$ and $E=8 \mu \mathrm{J}$, respectively. The pulse energy was controlled using the integrated attenuator. The laser source was integrated in a laser processing machine (microSTRUCT vario, 3D-Micromac, Chemnitz, Germany). A galvanometer scanner was used to scan the laser beam with the speed $v_{\text {scan }}$ across the sample. The focusing optic was a telecentric $f$-theta lens with a focal length of $100 \mathrm{~mm}$. Thus, a scan field size of $60 \times 60 \mathrm{~mm}^{2}$ was available. The focus radius 
was determined to be $w_{0} \approx 6 \mu \mathrm{m}$ according to Liu [19]; the Rayleigh length resulted to be about $200 \mu \mathrm{m}$. This configuration seemed to be a good compromise between minimal kerf widths and practicable focal depth. The polarization of the laser beam was oriented perpendicular to the scan direction for cutting the sides being bended in the bending test experiments, because numerous studies have already demonstrated that cuts become straighter and the surface quality of the sidewalls is higher under this condition [16, 20, 21]. It should be noted that the orientation of the polarization with respect to the scan direction changed as the chips were cut in the other two directions. However, since their cutting quality does not influence the bending test experiments, the orientation of the polarization was left unchanged.

In the first experiment of this study, the influence of fluence, pulse distance, and number of scans on the cutting quality was investigated. Thereby, it has to be taken into account that the fluence changes with the cutting depth, while the cutting quality depends on the fluence. During the first scan, the irradiated area is plane and the absorbed peak fluence $F_{\text {ap }}$ can be calculated as:

$F_{\text {ap }}=A_{\mathrm{p}} F_{0}=A_{\mathrm{p}} \frac{2 E}{w_{0}^{2} \pi}$,

where $F_{0}$ is the peak fluence of the Gaussian beam profile and $A_{\mathrm{p}}=0.63$ is the absorption of plane $\mathrm{Si}$ at a wavelength of $520 \mathrm{~nm}$ [22]. The formation of ever-steeper sidewalls during scribing increases continuously the irradiated area and the angle of incidence. The projected area on the sidewalls becomes elliptic and the absorbed fluence on the sidewall $F_{\text {as }}$ decreases. The length of the cut sidewall $w_{\mathrm{s}}$, which corresponds to the large axis of the projected ellipse, can be estimated in terms of the beam radius $w_{0}$ and the cutting depth $d$ using the Pythagorean theorem [17]. Besides, the steep sidewalls guide and trap reflected light inside the trench and thus increase absorption continuously up to $A_{\mathrm{s}}=1$. Ablation stops when the absorbed fluence on the sidewall $F_{\text {as }}$ reaches the ablation threshold fluence $F_{\text {th }}[5,17,23,24]$. The ablation threshold fluence $F_{\text {th }}$ is defined the minimum energy density to initiate material removal. The absorbed fluence on the sidewall as function of the cutting depth can be estimated as:

$$
F_{\mathrm{as}}=A_{\mathrm{s}} \frac{2 E}{w_{\mathrm{s}} w_{0} \pi}=A_{\mathrm{s}} \frac{2 E}{\sqrt{d^{2}+w_{0}^{2}} w_{0} \pi}=A_{\mathrm{s}} F_{0} \frac{1}{\sqrt{\frac{d^{2}}{w_{0}^{2}}+1}} .
$$

Recent investigations showed that the surface roughness depends strongly on the applied fluence [25]. Low fluences enhance the formation of laser-induced periodic surface structures (LIPSS) and high fluences increase melt ejections [26]. The optimal peak fluence for the ablation of plane silicon is located in the middle of these two regimes at about $F_{0}=2.8 \mathrm{~J} / \mathrm{cm}^{2}$ [25]. Inserting this value in Eq. (1) yields that the absorbed peak fluence should be $F_{\text {ap }}=1.8 \mathrm{~J} /$ $\mathrm{cm}^{2}$. The peak fluence $F_{0}$ that yields to an optimal fluence on the sidewall of a cut with the depth $d$ can be calculated by solving Eq. (2) for $F_{0}$ and inserting $F_{\text {as }}=F_{\text {ap }}=1.8 \mathrm{~J} / \mathrm{cm}^{2}$. This calculation suggests that $F_{0}$ should be set to $14.9 \mathrm{~J} / \mathrm{cm}^{2}$ to cut a wafer with a depth of $50 \mu \mathrm{m}$. In this study, the focus radius was fixed to $w_{0}=6 \mu \mathrm{m}$. The fluence was varied by changing pulse energy. A maximum fluence of $F_{0}=14.2 \mathrm{~J} /$ $\mathrm{cm}^{2}$ was available, which is very close to the "optimal fluence", while a fluence of about $6 \mathrm{~J} / \mathrm{cm}^{2}$ was just sufficient to completely cut the wafer. Thus, the fluence was varied between these values.

The pulse distance is another factor influencing the cutting quality. It should be large enough to avoid thermal heating and directed material ejections in the trench [17], but small enough to ablate a closed line when the beam is translated across the sample. Recent studies on surface ablation demonstrated that the highest ablation quality can be achieved if the ratio between the pulse distance and the focus radius is set to $d_{\mathrm{p}} / w_{0}=v_{\text {scan }} /\left(f_{\text {rep }} w_{0}\right) \approx 1[27,28]$. This criterion could be fulfilled with the present setup at a scan speed of $v_{\text {scan }}=1200 \mathrm{~mm} / \mathrm{s}$. The scan speed was varied around this optimal value between 100 and $2000 \mathrm{~mm} / \mathrm{s}$.

More precisely, the breaking strength is proportional to the quotient of the average fluence $F$ and the pulse distance $d_{\mathrm{p}}$ [15]. Thus, the wafers were also scribed in this study using different combinations of pulse energies and scan speeds, and the breaking strength was evaluated as function of the average accumulated fluence per scan, which calculates to:

$F_{\text {acc }}=F \frac{2 w_{0}}{d_{\mathrm{p}}}=\frac{E}{w_{0}^{2} \pi} \frac{2 w_{0} f_{\text {rep }}}{v_{\text {scan }}}=\frac{2 E f_{\text {rep }}}{w_{0} \pi v_{\text {scan }}}$.

The number of scans determines the cutting depth and also influences the breaking strength [18]. If the number of scans is too small, the wafer is only perforated $[18,20]$. However, if it is larger than necessary to separate the wafer, the sidewalls can be polished and the breaking strength of the backside increases [18]. In the present study, the maximum number of scans was selected such that the wafers were cut completely but the dicing tape below was still holding together. This made handling and shipping of the wafers much easier, but the selected numbers of scans might not have been optimal.

In a second experiment, different scanning strategies were used to optimize the backside breaking strength. Instead of the standard single line cut used in the first experiment, parallel lines were scanned and a step cut was generated. These principles are sketched in Fig. 1a-c. The parallel line cut was machined by scanning the beam in 


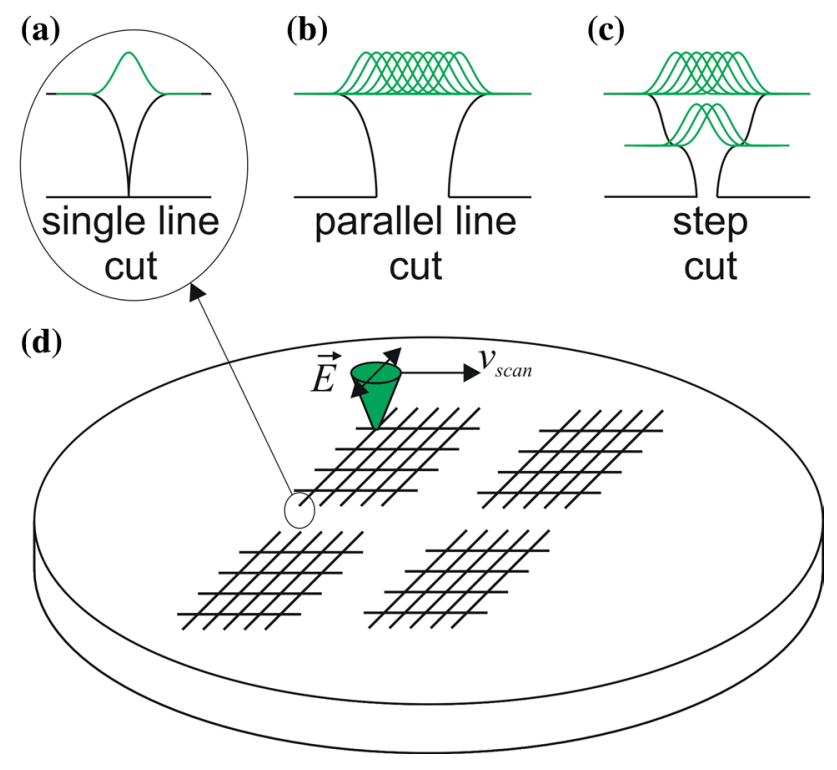

Fig. 1 Laser dicing strategies. Single line cuts (a) were generated in the first and parallel line cuts (b) and step cuts (c) in the second experiment. The green curves represent the intensity distribution of the beams and the black lines the cross section of the resulting cut. d $4 \times 247$ Chips were cut out of an 8 " wafer. The laser beam was scanned $N$ times with the speed $v_{\text {scan }}$ across the sample. The polarization was oriented perpendicular to the scan direction in horizontal direction

ten parallel lines with a distance of $5 \mu \mathrm{m}$ across the sample. This scan procedure was repeated until the wafer was cut completely. The idea of this strategy was to generate wider trenches to make picking easier, to minimize the risk of remaining bridges in the cut, to change the material ejection conditions, and to change the reflections and interference conditions. The idea of the step cut was to thin the waver to half its thickness within a wide groove, and to separate the lower half by a thinner cut to minimize the effect of decreasing fluence inside a deep trench. The first step was generated by translating the beam with a scan speed of $v=1 \mathrm{~m} / \mathrm{s}$ in $L=7$ parallel lines and $N=35$ repetitions across the wafer. The fluence was set to $7 \mathrm{~J} /$ $\mathrm{cm}^{2}$. The second cut was generated using $L=1$ or $L=3$ lines, scan speeds between $v=100 \mathrm{~m} / \mathrm{s}$ and $v=1000 \mathrm{~m} / \mathrm{s}$, and fluences between 3.5 and $14.2 \mathrm{~J} / \mathrm{cm}^{2}$. The number of repetitions $N$ was adjusted in the same way as in the first experiment. In this way, the step was located in the neutral axis of the wafer, which means that it had no influence on the bending test experiments. Moreover, the lower half of the wafer can be regarded as a separate $30 \mu \mathrm{m}$ thin wafer. This has the following consequences. The remaining half of the wafer can be cut with less pulse energy, because the cutting depth scales with the pulse energy [17]. In addition, the ratio between spot radius $w_{0}$ and wafer thickness $d$ changes, and thus the ratio between the absorbed fluences on the plain surface at the first scan $F_{\text {ap }}$ and on the sidewall at the final scan $F_{\text {as }}$ decreases.

The chips for mechanical testing were prepared by dividing a wafer in four quadrants and cutting a matrix of $13 \times 19$ chips per quadrant and parameter set (see Fig. 1d). In this way, a safety distance of about $5 \mathrm{~mm}$ to the edges of the maximum scan field size was maintained to ensure homogeneous energy distribution in the processed area. The cut chips were picked always in the same order by starting from one of the corners and removing them line by line. In this way, chip location-dependent effects should have similar contribution to the statistic in the bending-test experiments. It should be mentioned here that influences of the chip location were not recognized in the bending test results. Due to the small chip size, special care had to be taken during picking and placing the chips. Picking was particularly difficult because the chips had to be separated from the dicing tape. If the polymer tape was not cut, the chips were lifted from the tape with a small knife. Special care was taken that the knife edge did not touch the sides being bended during mechanical testing. For this purpose, chips were only picked from every second line and the chips in between were sacrificed. In the case that the polymer tape stuck to the backside of the chip, it was peeled off carefully with the finger nail. The chips were then placed by hand in a bending test machine.

A custom build three-point bending test setup was used in this study. This is a common method to test the mechanical strength of separated dies, and has thus already been used in several studies $[6,8,10,18,29-31]$. The test procedure was as follows: chips were placed on two supports with a distance of $L=2 \mathrm{~mm}$, a loading edge was lowered from the top until the chip was broken, and the breaking force $F_{\mathrm{b}}$ was measured. The flexural stress at break is:

$\sigma_{\mathrm{fb}}=\frac{3 F_{\mathrm{b}} L}{2 b h^{2}}(\mathrm{MPa})$

where $b$ and $h$ are the length and height of the sample, respectively. The bending test causes compressive stress at the load side and tensile stress at the support side. Because breaking of the chips is rather caused by tensile than by compressive stress, the back or front-side strength can be tested by placing the chips with the laser exit (Fig. 2a) or entrance side (Fig. 2b) on the two supports, respectively. The breaking strength of 30 chips was measured for each orientation, and the arithmetic mean and standard deviation was calculated. The bending tests were performed at room temperature.

\section{Results}

In the first experiment, wafers were separated using the single line cut and different combinations of pulse 

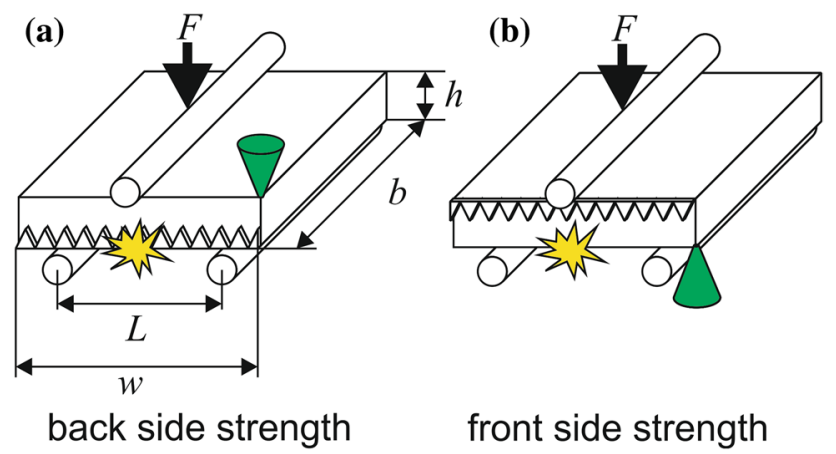

front side strength

Fig. 2 Mechanical testing of the cut chips. The backside (a) and front-side strength (b) of the cut chips were measured using a threepoint bending test setup. The distance between the two supports at the bottom was $L=2 \mathrm{~mm}$. The force $F$ was applied from the top. The chip dimensions were $h \approx 60 \mu \mathrm{m}, w \approx 3 \mathrm{~mm}, b \approx 4 \mathrm{~mm}$

energies, scan speeds, and numbers of scans. The frontand back-side strength of the cut chips are measured and plotted as function of the average accumulated fluence per scan in Fig. 3.

The results showed that the front-side breaking strength decreased with increasing accumulated fluence per scan. It was also observed that both higher scan speeds and lower pulse energies lead to higher breaking strengths. These findings are in well agreement with the results achieved by Finn et al. [15].

Regarding the back-side breaking strength, the trend of fitted curve indicates that the accumulated fluence per scan has also a slight influence on the mechanical stability. However, the level of the backside breaking strength was significantly lower than the front-side breaking strength. It dropped from about $1000 \mathrm{MPa}$ to only $400 \mathrm{MPa}$. The variation of pulse energy, number of scans and scan speed showed no significant improvement of the backside breaking strength. Consequently, if it is possible to improve the breaking strength, there must be other or additional parameters taken into consideration to improve the situation.

In a second experiment, the wafers were cut by scribing parallel lines and by applying the step cut to improve the backside breaking strength. Besides, the pulse energy and the number of scan $N$ were varied. The backside breaking strength was measured and plotted as function of the peak fluence $F_{0}$ in Fig. 4. For comparison, the best results using single line cuts with a fluence of $F_{0}=8 \mathrm{~J} / \mathrm{cm}^{2}$, which was the minimum fluence to cut the wafer, and the maximum fluence available of $F_{0}=14.2 \mathrm{~J} / \mathrm{cm}^{2}$ were plotted in the graph.

The parallel line cuts were performed using different combinations of pulse energies and numbers of scans. However, it was not possible to achieve breaking strengths higher than $600 \mathrm{MPa}$. These findings indicate that widening of the trench does not improve the backside breaking strength significantly.

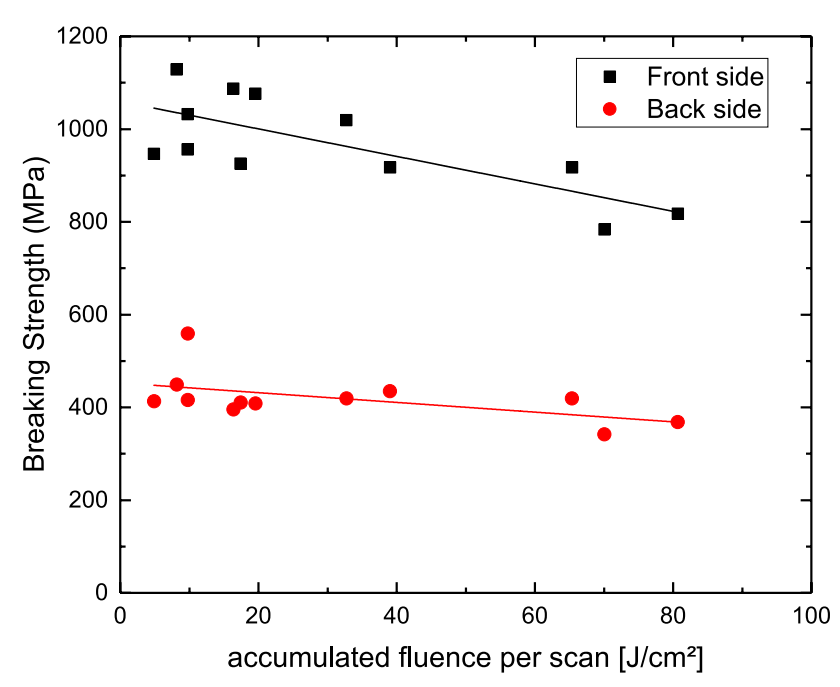

Fig. 3 Front (black squares) and back-side (red dots) breaking strength as function of the accumulated fluence per scan. The straight lines represent least square linear fits to the data points

Remarkably, the breaking strength increased significantly as the step cut strategy was used. The maximum breaking strength of about $1187 \mathrm{MPa}$ was achieved when the fluence was set to $F_{0}=4 \mathrm{~J} / \mathrm{cm}^{2}$. At lower fluences, the wafer was only perforated and the breaking strength dropped to $500 \mathrm{MPa}$. The lower step was cut also by scanning a single line instead of three parallel lines, but the breaking strength remained on the same level (compare green and blue triangle at about $7 \mathrm{~J} /$ $\mathrm{cm}^{2}$ in Fig. 4). These results demonstrate that the mechanical strength of the front- and backside of fs-laser cut silicon wafers can be on the same high level. Besides fluence, scan speed, and number of scans, the choice of the right scan

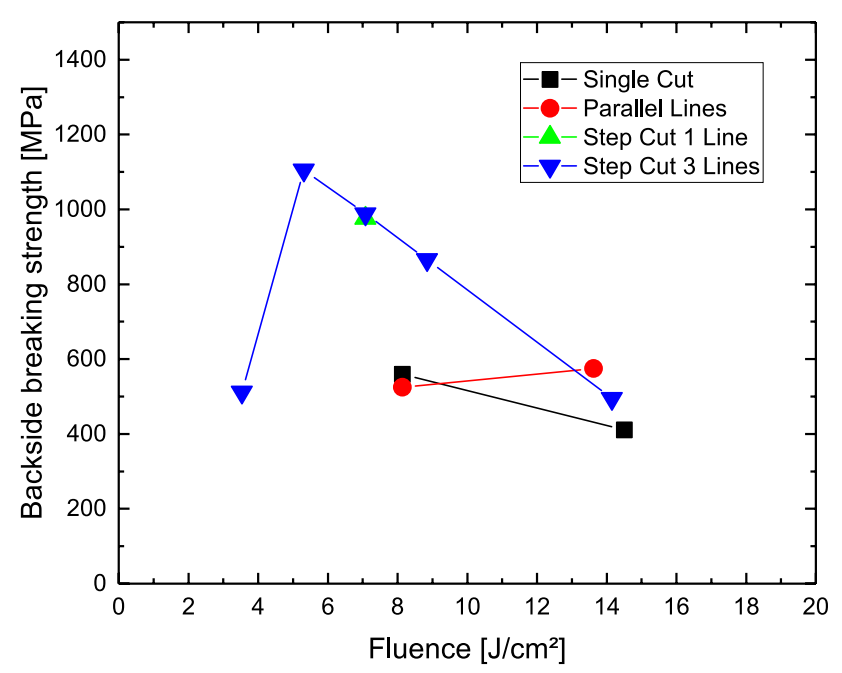

Fig. 4 Mean backside breaking strength using different cutting strategies 
strategy plays a crucial role in laser dicing. In particular, the ratio between the beam radius and the wafer thickness could be important.

The best results achieved for the front-side (left diagram) and backside (right diagram) breaking strength using fslaser dicing were compared to results achieved by mechanical and ns-laser dicing on state-of-the-art dicing machines with already optimized parameter settings. The breaking strength of the chips is plotted as Weibull distributions in Fig. 5. The average values of the frontside breaking strengths shown in the left diagram were $931 \pm 110,390 \pm 56$, and $1129 \pm 98 \mathrm{MPa}$ for the fs-laser, ns-laser, and mechanically diced chips, respectively. The average values of the backside breaking strengths shown in the right diagram were $968 \pm 235,781 \pm 173$, and $1105 \pm 210 \mathrm{MPa}$, respectively. This comparison revealed that both the highest front and the highest backside breaking strength could be achieved using the fs-laser. The ns-laser showed in both cases the worst performance. Moreover, the slope of the fs-laser diced chips is the steepest, indicating that the reliability could be improved compared to mechanical dicing, which is also an important property for industrial manufacturing.

\section{Discussion}

The aim of this study was to find strategies to improve the breaking strength of ultrafast laser-cut thin silicon wafers at both the laser entrance and exit side. The results suggest that the ultrafast laser has the potential to outperform the wafer saw if the right combinations of laser parameters are chosen.

Regarding the mechanical stability of the cut dies at the laser entrance side, the maximum average die breaking strength was measured to be in the order of $1100 \mathrm{MPa}$ in this study. The results suggest that the key to improve the cutting quality is to minimize the accumulated fluence per scan. This finding is in well agreement with the result of Finn et al. [15]. Surprisingly, the highest breaking strength values measured in this study are even slightly higher than the best results presented by Finn et al. [15], although they used a laser system with a shorter wavelength of $355 \mathrm{~nm}$. Because the optical penetration depth in $\mathrm{Si}$ is shorter at $355 \mathrm{~nm}$ than at $520 \mathrm{~nm}$, the heat-affected zone should be smaller and thus the breaking strength higher. Since the opposite was observed, a possible explanation could be that the absolute breaking strength may also depend on the setup with which it was measured. Nevertheless, the fact that the breaking strength using 520 and $355 \mathrm{~nm}$ is on the same level leads to the question, if cutting with a UV-laser is necessary. Future experiments should be carried out if the cutting quality really benefits from the higher conversion losses and higher costs of UV-lasers.

A similar relation between accumulated fluence per scan and breaking strength could be also observed for the backside. However, the level of the average backside breaking strength was typically in the order of about $400 \mathrm{MPa}$, which is more than a factor of two lower than the front-side breaking strength. These findings are in well agreement with the results of Haupt et al. [10]. Remarkably, the step cut increased the average breaking strength to above $1100 \mathrm{MPa}$, which is at the same level as the best results for the front-side breaking strength. This leads to the question why the step cut led to such an enormous improvement. Therefore, the geometry of the cuts has to be considered. The step cut was a wide trench reducing the thickness to about $d=30 \mu \mathrm{m}$, followed by a
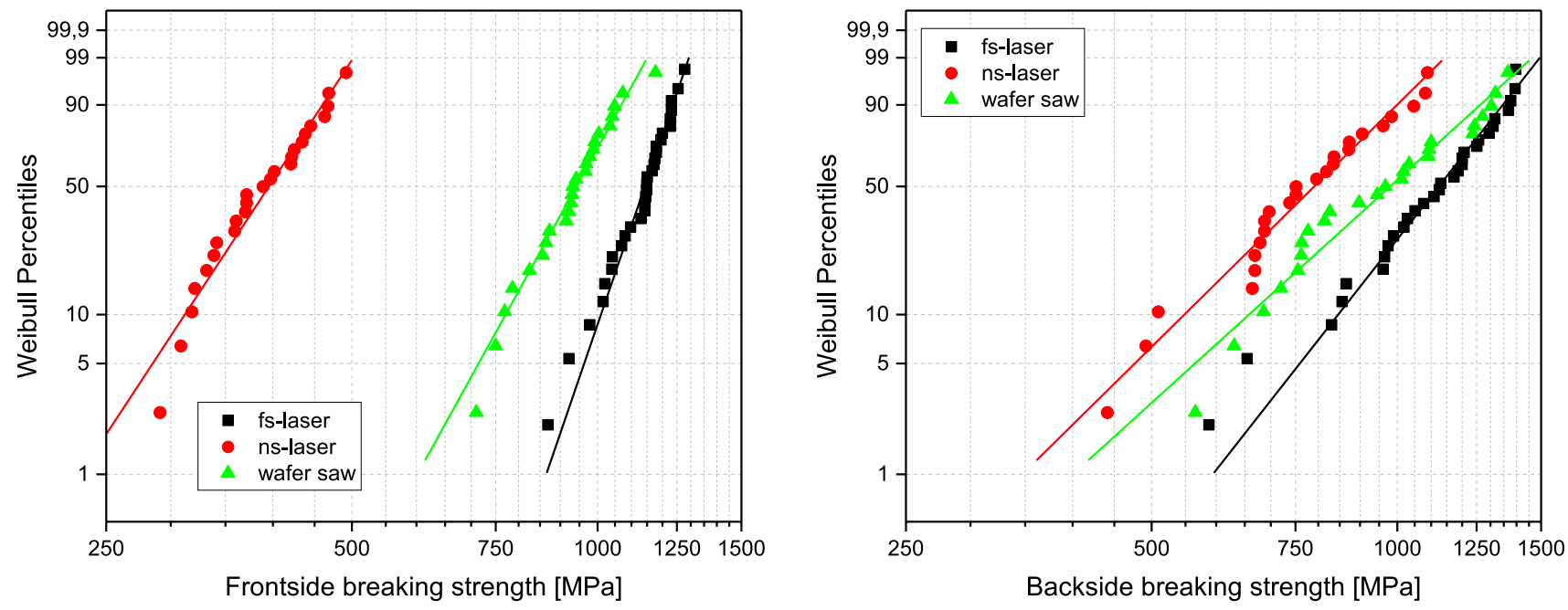

Fig. 5 Weibull distributions of the front-side (left diagram) and backside (right diagram) breaking strength achieved with fs-laser, ns-laser, and mechanical (wafer saw) dicing. The data points for fs-laser dicing belong to the best results achieved in this study 
smaller cut in the thinned wafer. Because the step was in the neutral axis of the wafer, fabricating the second step could be regarded as cutting a thinner wafer. This could have had the following consequences. As Fig. 4 revealed, the best results were achieved at fluences of $F_{0}=5.3 \mathrm{~J} /$ $\mathrm{cm}^{2}$, which is well below the minimum fluence needed to cut through the full wafer thickness. Solving Eqs. (1) and (2) for a thickness $d=30 \mu \mathrm{m}$ yields absorbed fluences during the first and last scan $F_{\text {ap }}=3.3 \mathrm{~J} / \mathrm{cm}^{2}$ and $F_{\text {as }}=1.0 \mathrm{~J} / \mathrm{cm}^{2}$, respectively. These fluence values are in the range of the optimal fluence for surface ablation of $1.8 \mathrm{~J} / \mathrm{cm}^{2}[25,28]$. This calculation suggests that the fluence could have been low enough to reduce melt ejections [26] as well as periodic hole and kerf formations [17] during the first scans, but still high enough to suppress enhanced LIPSS formation during the last scans [26]. In simple terms, the step cut could have made it possible to use an optimal fluence ratio. Other reasons for the improved breaking strength could be related to the shallower taper angle. On the one hand, this could improve debris ejection, on the other hand, the sidewall polishing effect reported in [18] could be more pronounced. Because the ratio between beam radius $w_{0}$ and thickness $d$ plays an important role in all those scenarios, future experiments will be performed using larger beam radii. The results further indicate that there is a trade-off between minimum kerf width and mechanical stability. Nevertheless, it should be pointed out that larger kerf widths of about $40 \mu \mathrm{m}$ are rather desired for industrial production, because this allows safer picking and placing.

As mentioned in the introduction, the current challenge for ultrafast-laser dicing of thin Si wafers is to improve both the mechanical strength and the cutting speed in comparison with mechanical dicing with a wafer saw. Even though several studies were carried out before, this is the first time to the knowledge of the authors that a process window was found that enables to cut thin silicon wafers with higher quality at the front and backside than with a wafer saw. However, these results were achieved only at effective cutting speeds of $v_{\text {scan }} / N=1.45 \mathrm{~mm} / \mathrm{s}$ and laser powers of $0.67 \mathrm{~W}$. This yields to specific cutting speeds of about $2.2 \mathrm{~mm} / \mathrm{s} / \mathrm{W}$. Dividing the cutting speed of the wafer saw, which is about $100 \mathrm{~mm} / \mathrm{s}$, by this specific cutting speed indicates that an ultrashort pulse laser with a power of about $45 \mathrm{~W}$ would be necessary to achieve speeds, which are state of the art in semiconductor industry. Because the results of this study suggest that the backside breaking strength depends on pulse energy and beam radius, power scaling is mainly possible by increasing the pulse frequency or by parallel processing. Since higher repetition frequencies could increase thermal side effects, future experiments should address the impact of high repetition rates on mechanical stability.

\section{Conclusion}

Recent investigations showed that ultrafast-laser dicing increases the mechanical stability of the cut chips compared to mechanical dicing, however only at the front and not at the backside. The results of this study revealed for the first time to the knowledge of the authors that it is indeed possible to improve also the backside breaking strength using ultrafast lasers. Maximum average breaking strengths of about 1128 and $1105 \mathrm{MPa}$ were achieved for front and backside, respectively. These values were even higher than typical breaking strengths obtained using nslaser (389 and $781 \mathrm{MPa}$ ) and mechanical dicing (931 and $968 \mathrm{MPa}$ ) machines with already optimized parameters.

Cutting experiments using a single line scanning strategy showed that the breaking strength increases on both sides with decreasing accumulated fluence per scan. However, the backside breaking strength was always below $600 \mathrm{MPa}$. Experiments with different scan strategies revealed that a step cut improves the backside breaking strength to about $1100 \mathrm{MPa}$. An explanation for this improvement could be found by calculating the laser fluence values during the first scan on the plain surface and during the last scan on the sidewall. Because the step cut thinned the waver to half its thickness within a wide groove, the pulse energy could be reduced to cut the lower half and the fluence absorbed by the sidewalls remained at an optimal level between 3.3 and $1.0 \mathrm{~J} / \mathrm{cm}^{2}$. At this fluence regime, ablation efficiency is optimal, and melt ejections, heat-induced side effects, periodic holes, and LIPSS are minimal. In addition, the larger kerfs generated by the step cut could enable more efficient debris ejection or could be better suited for sidewall polishing by continuing scanning the beam across the wafer even after separation.

Because the conditions described in the paragraph before can be also fulfilled using larger beam radii or by ramping pulse energy during scribing, future experiments will be carried out to optimize the dicing strategy further and to improve cutting speed. Nevertheless, the results of this study already demonstrated that ultrafast-laser dicing has the great potential to outperform state-of-the-art dicing methods and to achieve chips with unprecedented mechanical stability in chip mass production.

Acknowledgements Open access funding provided by FH Vorarlberg-University of Applied Sciences. Thanks to Tobias Polster for performing the dicing experiments. Thanks to all colleagues at the research center for microtechnology at the Vorarlberg University of Applied Sciences for the technical support and the interesting discussions. The financial support by the Austrian Federal Ministry of Science, Research and Economy and the National Foundation for Research, Technology and Development is gratefully acknowledged.

Open Access This article is distributed under the terms of the Creative Commons Attribution 4.0 International License (http:// 
creativecommons.org/licenses/by/4.0/), which permits unrestricted use, distribution, and reproduction in any medium, provided you give appropriate credit to the original author(s) and the source, provide a link to the Creative Commons license, and indicate if changes were made.

\section{References}

1. J.U. Knickerbocker, P.S. Andry, B. Dang, R.R. Horton, M.J. Interrante, C.S. Patel, R.J. Polastre, K. Sakuma, R. Sirdeshmukh, E.J. Sprogis, S.M. Sri-Jayantha, A.M. Stephens, A.W. Topol, C.K. Tsang, B.C. Webb, S.L. Wright, Three-dimensional silicon integration. IBM J. Res. Dev. 52(6), 553-569 (2008)

2. J.H. Lau, Overview and outlook of through-silicon via (TSV) and 3D integrations. Microelectron. Int. 28(2), 8-22 (2011)

3. Tan Zhang, Zhenwei Hou, R.W. Johnson, L. Del Castillo, A. Moussessian, R. Greenwell, B.J. Blalock, Flexible electronics. IEEE Trans. Electron. Packag. Manuf. 32(4), 291-300 (2009)

4. Y.-H. Zhang, S. Karthikeyan, J. Zhang, Polymer-sandwich ultrathin silicon(100) platform for flexible electronics. Chin. Phys. Lett. 33(6), 66201 (2016)

5. C. Fornaroli, J. Holtkamp, A. Gillner, Dicing of thin Si wafers with a picosecond laser ablation process. Phys. Proc. 41, 603-609 (2013)

6. B. Majeed, I. Paul, K.M. Razeeb, J. Barton, S.C. O'Mathuna, Microstructural, mechanical, fractural and electrical characterization of thinned and singulated silicon test die. J. Micromech. Microeng. 16(8), 1519-1529 (2006)

7. S.-H. Chae, J.-H. Zhao, D.R. Edwards, P.S. Ho, Effect of dicing technique on the fracture strength of Si dies with emphasis on multimodal failure distribution. IEEE Trans. Device Mater. Relib. 10(1), 149-156 (2010)

8. N. Sudani, K. Venkatakrishnan, B. Tan, Laser singulation of thin wafer: Die strength and surface roughness analysis of $80 \mu \mathrm{m}$ silicon dice. Opt. Lasers Eng. 47(7-8), 850-854 (2009)

9. A. Ostendorf, C. Kulik, T. Bauer, N. Baersch, Ablation of metals and semiconductors with ultrashort-pulsed lasers: improving surface qualities of microcuts and grooves. Proc. SPIE 5340, 153-163 (2004)

10. O. Haupt, F. Siegel, A. Schoonderbeek, L. Richter, R. Kling, A. Ostendorf, Laser dicing of silicon: comparison of ablation mechanisms with a novel technology of thermally induced stress. J. Laser Micro Nanoeng. 3(3), 135-140 (2008)

11. M. Koitzsch, M. Schellenberger, in Thermal Laser Separation (TLS) for Separating Multi-Crystalline Silicon Wafers: A Comparison with State-of-the-Art Methods. 26th European Photovoltaic Solar Energy Conference (2011)

12. M. Kumagai, N. Uchiyama, E. Ohmura, R. Sugiura, K. Atsumi, K. Fukumitsu, Advanced dicing technology for semiconductor wafer-stealth dicing. IEEE Trans. Semicond. Manuf. 20(3), 259-265 (2007)

13. P.C. Verburg, G.R.B.E. Römer, A.J. Huis in 't Veld, Two-photoninduced internal modification of silicon by erbium-doped fiber laser. Opt. Express 22(18), 21958-21971 (2014)
14. B.N. Chichkov, C. Momma, S. Nolte, F. von Alvensleben, A. Tuennermann, Femtosecond, picosecond and nanosecond laser ablation of solids. Appl. Phys. A 63(2), 109-115 (1997)

15. D.S. Finn, Z. Lin, J. Kleinert, M.J. Darwin, H. Zhang, Study of die break strength and heat-affected zone for laser processing of thin silicon wafers. J. Laser Appl. 27(3), 32004 (2015)

16. T.H.R. Crawford, A. Borowiec, H.K. Haugen, Femtosecond laser micromachining of grooves in silicon with $800 \mathrm{~nm}$ pulses". Appl. Phys. A 80(8), 1717-1724 (2005)

17. M. Domke, B. Egle, G. Piredda, G. Fasching, M. Bodea, E. Schwarz, Controlling depth and distance of the hole formations at the bottom of laser-scribed trenches in silicon using fs-pulses. Proc. SPIE 93501J, 1-6 (2015)

18. M. Domke, B. Egle, G. Piredda, S. Stroj, G. Fasching, M. Bodea, E. Schwarz, Ultrashort pulse laser dicing of thin Si wafers. J. Micromech. Microeng. 26(11), 115004 (2016)

19. J.M. Liu, Simple technique for measurements of pulsed Gaussianbeam spot sizes. Opt. Lett. 7(5), 196-198 (1982)

20. N. Bärsch, K. Körber, A. Ostendorf, K.H. Tönshoff, Ablation and cutting of planar silicon devices using femtosecond laser pulses. Appl. Phys. A 77(2), 237-242 (2003)

21. H.K. Tönshoff, A. Ostendorf, T. Wagner, Structuring silicon with femtosecond lasers. Proc. SPIE 4274, 88-97 (2001)

22. M.A. Green, M.J. Keevers, Optical properties of intrinsic silicon at 300 K. Prog. Photovolt.: Res. Appl. 3(3), 189-192 (1995)

23. C. Fornaroli, J. Holtkamp, A. Gillner, in Dicing of Thin Silicon Wafers with Ultra-Short Pulsed Lasers in the Range from $200 \mathrm{fs}$ up to 10 ps. Proceedings of LPM2014 (2014)

24. C. Fornaroli, J. Holtkamp, A. Gillner, Dicing of thin Si wafers with a picosecond laser ablation process. Proc. ICALEO M201, $1-5$ (2013)

25. M. Domke, G. Piredda, J. Zehetner, S. Stroj, Minimizing the surface roughness for silicon ablation with ultrashort laser pulses. JLMN 11(1), 100-103 (2016)

26. M. Domke, Conditions for random and periodic hole formations during surface ablation of silicon with ultrashort pulsed lasers. Proc. SPIE 935511, 1-7 (2015)

27. B. Neuenschwander, G.F. Bucher, G. Hennig, C. Nussbaum, B. Joss, M. Muralt, S. Zehnder, U.W. Hunziker, P. Schütz, Processing of dielectric materials and metals with ps laserpulses. Proc. ICALEO 707-715 (2010)

28. M. Domke, G. Piredda, S. Stroj, Calculating the optimal combination of pulse-to-pulse distance and fluence for scribing and patterning with ultrashort pulsed lasers. Proc. LIM 1-6 (2015)

29. D.Y.R. Chong, W.E. Lee, B.K. Lim, J.H.L. Pang, T.H. Low, Mechanical characterization in failure strength of silicon dice. Proc. ITHERM 2, 203-210 (2004)

30. S. Schoenfelder, M. Ebert, C. Landesberger, K. Bock, J. Bagdahn, Investigations of the influence of dicing techniques on the strength properties of thin silicon. Microelectron. Reliab. 47(2-3), 168-178 (2007)

31. D.A. Porter, T.A. Berfield, Die separation and rupture strength for deep reactive ion etched silicon wafers. J. Micromech. Microeng. 23(8), 85020 (2013) 